\title{
Dynamic Viscoelastic Behavior of Model Poly(vinyl chloride) Blends Characterized at Small Deformation in Shear Oscillation
}

\author{
Seung-Yeop KwaK* \\ Institute of Polymer Engineering, The University of Akron, \\ Akron, Ohio 44325-0301, U.S.A.
}

(Received October 8, 1993)

\begin{abstract}
In the present study, dynamic shear oscillation measurements at small strain were conducted on four model blends of equal amounts (50/50 by weight) of poly(vinyl chloride) (PVC) with three compatible and one incompatible polymers. The compatible polymers were nitrile rubber containing 30\% acrylonitrile (NBR-30), poly(E-caprolactone) (PCL), and copolyester thermoplastic elastomer (trademark HYTREL). The incompatible one was nitrile rubber containing $22 \%$ acrylonitrile (NBR-22). Double logarithmic plots of loss modulus $G^{\prime \prime}(\omega)$ against storage modulus $G^{\prime}(\omega)$ were employed as a systematic, practical way of treating the data. The $\log G^{\prime \prime}(\omega)-\log G^{\prime}(\omega)$ scheme was shown to enable one to characterize and interpret the viscoelastic behavior of the blends related to the mixing history and morphological changes.
\end{abstract}

KEY WORDS Poly(vinyl Chloride) (PVC) / Nitrile Rubber (NBR) / Poly( $\varepsilon$-caprolactone) (PCL) / Copolyester Thermoplastic Elastomer (HYTREL) / Mechanical Blending / Viscoelastic Behavior / Shear Storage Modulus $G^{\prime}(\omega)$ / Shear Loss Modulus $G^{\prime \prime}(\omega) / \log G^{\prime \prime}(\omega)-\log G^{\prime}(\omega)$ Plot /

Dynamic mechanical measurements have been used for characterizing a wide range of polymeric materials. Common features of the dynamic mechanical measurements are to impose a periodic, uniform sinusoidal deformation on a specimen and then observe the storage (elastic) and loss (viscous) moduli of the specimen.

Dynamic mechanical analysis may be classified into two categories; the first is associated with the measurements involving free vibrations and in the second are those based on forced oscillations. ${ }^{2,3}$ Among those falling in the second category, the dynamic shear oscillation measurement at small strain has been used to not only characterize the viscoelastic properties but also relate them to identify the differences in molecular structure and predict the behavior during processing. It is thus one of the most powerful techniques for studying the effect of not only molecular structure but also phase morphology on the physical properties and determining the processability and product performance, both of which are essential in component design.

Among the dynamic viscoelastic parameters measured at small strain in shear oscillation, the shear storage modulus $G^{\prime}$ and shear loss modulus $G^{\prime \prime}$ are of typical importance especially when elastic and viscous responses are needed to be rigorously separated. Both the storage and loss moduli can be obtained either as a function of temperature (at constant frequency; isochronal) or as a function of frequency (at constant temperature; isothermal). The storage and loss moduli as a function of frequency are presented as $G^{\prime}(\omega)$ and $G^{\prime \prime}(\omega)$, respectively. The interpretation of the meaning

* Present address: Division of Polymer Research, Korea Institute of Science and Technology (KIST), P. O. Box 131, Cheongryang, Seoul 130-650, Korea. 
of individual $G^{\prime}(\omega)$ and $G^{\prime \prime}(\omega)$ curves are useful in obtaining information of the viscoelastic behavior of a given material. A more practical and systematic way to have a better understanding and utilization of the $G^{\prime}(\omega)$ and $G^{\prime \prime}(\omega)$ data in conjunction with the molecular structure and phase morphology as well as the processing characteristics of the given material is to plot a double logarithmic form of $G^{\prime \prime}(\omega)$ against $G^{\prime}(\omega),{ }^{4}$ in the analogy to "Cole-Cole plot". 5 The Cole-Cole plot was formulated in a linear form to analyze and represent complex dielectric constant data, and has been adapted by other investigators ${ }^{6-9}$ to perform similar analysis of the viscoelastic data of polymeric materials. The $\log G^{\prime \prime}(\omega)-\log G^{\prime}(\omega)$ data presentation is a mapping of the relative contribution of $G^{\prime \prime}(\omega)$ to that of $G^{\prime}(\omega)$ and is appropriate for the polymeric materials usually having a broad distribution of relaxation times.

The $\log G^{\prime \prime}(\omega)-\log G^{\prime}(\omega)$ plot has been demonstrated for a number of homopolymers or copolymers as one of the effective, systematic means to analyze not only the molecular structure variations such as molecular weight (distribution), long branching and gels but also solution concentration dependence and morphology, which are well documented in the literature. ${ }^{4}$ However, with the $\log G^{\prime \prime}(\omega)$ $\log G^{\prime}(\omega)$ scheme, relatively little effort has been put on investigating the viscoelastic properties of polymer blends and relating them to the mixing history and phase morphology of the blends, which are essential from the practical point of view.

Therefore, it is the objective of the present study to perform the viscoelastic investigations of polymer blends using the $\log G^{\prime \prime}(\omega)-$ $\log G^{\prime}(\omega)$ scheme and then interpret them related to the mixing history and phase morphology. Chosen in this paper as model blends were four different systems of equal amounts of polyvinyl chloride (PVC) with three compatible and one incompatible polymers, whose systems have enjoyed commercial success and/or been of academic interest.
Compatible polymers were nitrile rubber (butadiene-acrylonitrile copolymer or NBR) containing $30 \%$ acrylonitrile (denoted as NBR-30), poly( $\varepsilon$-caprolactone) (PCL), and copolyester thermoplastic elastomer (trademark HYTREL). Incompatible one was nitrile rubber containing $22 \%$ acrylonitirle (NBR-22), which was selected in order to see the effect of incompatibility, relative to the compatible polymer.

\section{EXPERIMENTAL}

\section{Materials}

Poly(vinyl chloride) (PVC) resin was Geon 103 EP F-76, a product of B.F. Goodrich Co., with number-average molecular weight $\left(M_{n}\right)$ of 40,000 and weight-average molecular weight $\left(M_{w}\right)$ of $89,000 .^{10}$

Two grades of nitrile rubber (butadiene-acrylonitrile copolymer or NBR) were HYCAR VT 380 (acrylonitrile content 30\%) and HYCAR 1094-80 (acrylonitrile content 22\%), both from Zeon Chemicals. They are denoted as NBR-30 and NBR-22, respectively. It was informed from the manufacturer that the glass transition temperature $\left(T_{\mathrm{g}}\right)$ of NBR-30 is $-28^{\circ} \mathrm{C}$ and that of NBR-22 is $-40^{\circ} \mathrm{C}$.

Poly( $\varepsilon$-caprolactone) (PCL) supplied by Union Carbide Co. was PCL-700. Its melting point is about $60^{\circ} \mathrm{C}$ and $T_{\mathrm{g}}$ is about $-60^{\circ} \mathrm{C}$. It has been reported that $M_{n}$ and $M_{w}$ of PCL are 13,000 and 41,000, respectively. ${ }^{11}$

Copolyester thermoplastic elastomer with the trade name of HYTREL (grade 4056) was a product of E.I. du Pont de Nemours \& Co. HYTREL is a random block copolymer of crystallizable tetramethylene terephthalate (4GT), which forms the hard segments, and poly(tetramethylene ether) glycol terephthalate (PTMEGT), the soft segments. ${ }^{12}$ The soft segments have $T_{\mathrm{g}}$ of about $-50^{\circ} \mathrm{C}$ and the hard segments melt at about $150^{\circ} \mathrm{C} . M_{n}$ of HYTREL is about 30,000 . 
Mixing Procedures and Sample Preparation

All of the model blends (i.e., PVC/NBR-30, PVC/NBR-22, PVC/PCL, PVC/HYTREL) consisted of $50 \%$ (by weight) of PVC with $1 \%$ thermal stabilizer (Therm-Chek 1827 from Ferro Corp.) and $50 \%$ (by weight) of the other constituent polymer. The mixings were done in a batch, non-intermeshing, counter-rotating two-wing rotor, Banbury-type internal mixer (1.57 liter Farrel Banbury mixer), which is schematically shown in Figure 1. The rotors were turned with the rpm of 80 and the fill factor in the mixing chamber was around 0.7 , which is typical in the commercial operation. ${ }^{13}$

The details of the mixing procedures are as follows;

i) In the case of PVC/NBR-30 and PVC/NBR-22 systems, NBR was first masticated at room temperature for 30 seconds and then, the rotor was stopped. At this stage, the temperature indicator of the mixer indicated $65^{\circ} \mathrm{C}$.

In the case of PVC/PCL system, the temperature of the chamber wall was first set

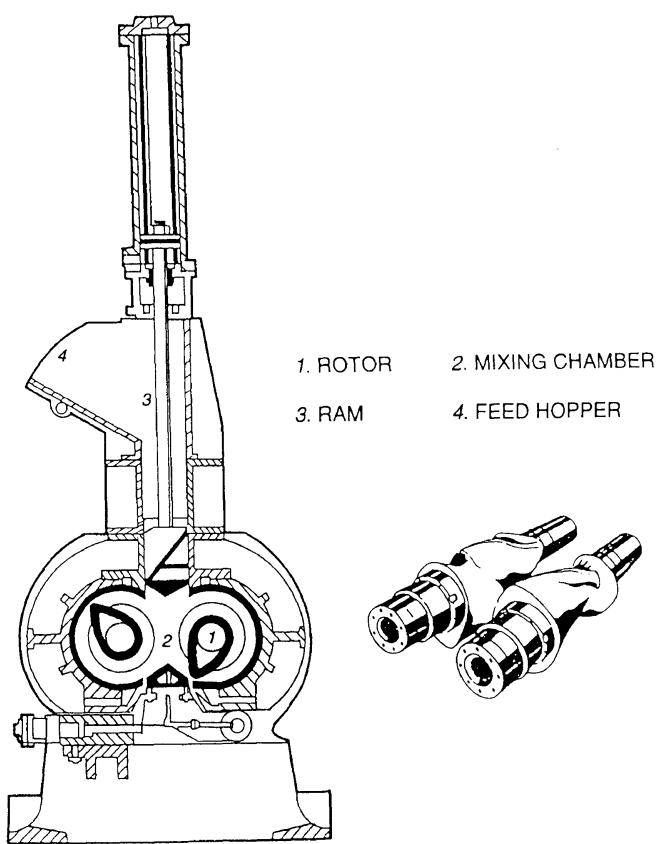

Figure 1. Schematic view of Banbury-type internal mixer. at $65^{\circ} \mathrm{C}$ and PCL was charged. Then, the rotor was rotated for 30 seconds in order for the complete melting of PCL.

For PVC/HYTREL system, the temperature of the chamber wall was first set at $135^{\circ} \mathrm{C}$ and then HYTREL was softened for 30 seconds.

ii) PVC was then charged in the mixer, the ram was lowered and the rotor was started to rotate. From the moment the rotor was started, the mixing times were measured and the whole mixing process was terminated in total 450 seconds. At 450 seconds, the mixing was considered to be completed. The observed temperature during mixing was shown to go over a maximum and then decrease slightly to a plateau. Figure 2 represents the schematic diagram of temperature changes during the mixing process. This diagram was obtained by monitoring the temperature changes appearing in the temperature indicator of the mixer. The reading in the indicator is the temperature of

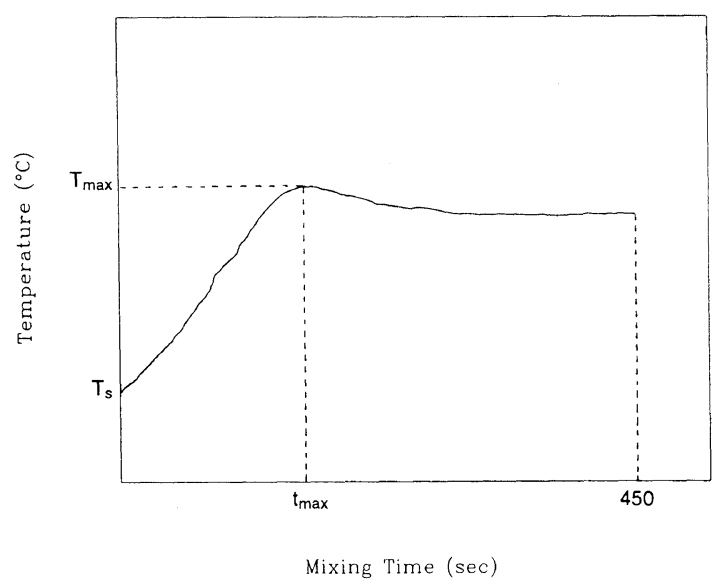

Figure 2. Schematic representation of temperature changes with progress of mixing.

Table I. Observed temperatures and times of mixings of all model blends

\begin{tabular}{lccc}
\hline Model Mixing & $T_{\mathrm{s}} /{ }^{\circ} \mathrm{C}$ & $T_{\max } /{ }^{\circ} \mathrm{C}$ & $t_{\max } / \mathrm{s}$ \\
\hline PVC/NBR-30 & 65 & 165 & 150 \\
PVC/NBR-22 & 65 & 165 & 150 \\
PVC/PCL & 65 & 160 & 130 \\
PVC/HYTREL & 135 & 175 & 110 \\
\hline
\end{tabular}


the surface of the mixing chamber detected by a thermocouple. In the figure, $T_{\mathrm{s}}$ is the temperature at which mixing started, $T_{\max }$ is the observed maximum temperature through the entire mixing process, and $t_{\max }$ is the mixing time corresponding to $T_{\max }$. Summarized in Table I are $T_{s}, T_{\max }$, and $t_{\max }$ of the individual mixing experiments of the model blends. It should be noted that since the temperature shown in Table I is that of the chamber surface detected by a thermocouple, the actual temperature of the material itself is somewhat higher than that of the chamber surface because of a very poor thermal conductivity of polymers.

After the completion of mixing, the content in the mixer was taken out and then sheeted out through a two-roll laboratory open mill in order to turn the large chunks into easily handled sheets. The sheeted-out samples were cut into small pieces for compression molding. The compression molding was done in a Wabash Compression Molding Press operated at $160^{\circ} \mathrm{C}$ for 150 minutes. While still hot, the compressed samples were taken out and cooled, being placed between two metal plates in order to provide a smooth surface. After cooling, circular shaped specimens with diameter of $25 \mathrm{~mm}$ were punched out for the dynamic mechanical measurements.

\section{Dynamic Mechanical Measurements-Frequency \\ Sweep}

The dynamic mechanical properties of the model blends were measured with the Rheometrics Mechanical Spectrometer (RMS) model RMS-800 in dynamic shear oscillatory mode using $25 \mathrm{~mm}$-diameter parallel discs. The range of angular frequency was from $10^{-1}$ to $10^{2} \mathrm{rad} \mathrm{s}^{-1}$.

Individual blend systems were tested within the temperature range where the sample stability is assured. Within this temperature range, neither the slippage between the surfaces of the discs and sample (due to lower temperatures) nor thermal degradation of the sample (due to higher temperatures) take place. In order to examine the sample stability, each sample was tested at the selected temperature from low frequency to high frequency (upsweep) and then tested immediately in the reverse direction (down-sweep) at the same temperature. The sample stability was assured whenever the reproducibility of the data between up-sweep and down-sweep was satisfactory. The strain amplitude was selected in the linear response of each sample with respect to the strain sweep at each test condition. The selected strain was not only large enough for accurate torque signals but also small enough to keep the material response in the linear region.

\section{RESULTS}

In Figures 3 and $4, G^{\prime}(\omega)$ and $G^{\prime \prime}(\omega)$ data of PVC/NBR-30 and PVC/NBR-22 blends at different temperatures are represented respectively in a double logarithmic form, forming separated plots as shown. Although the frequency does not appear in the plots, the lower values of $G^{\prime}(\omega)$ and $G^{\prime \prime}(\omega)$ data correspond to the lower frequency responses, and vice versa. The $45^{\circ}$ straight line indicates an equal-moduli line which corresponds to the

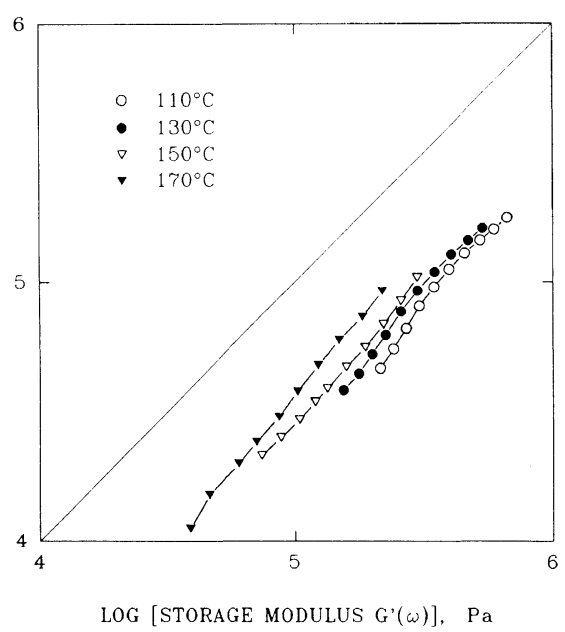

Figure 3. Plot of $\log G^{\prime \prime}(\omega)-\log G^{\prime}(\omega)$ for PVC/NBR-30 blend. 


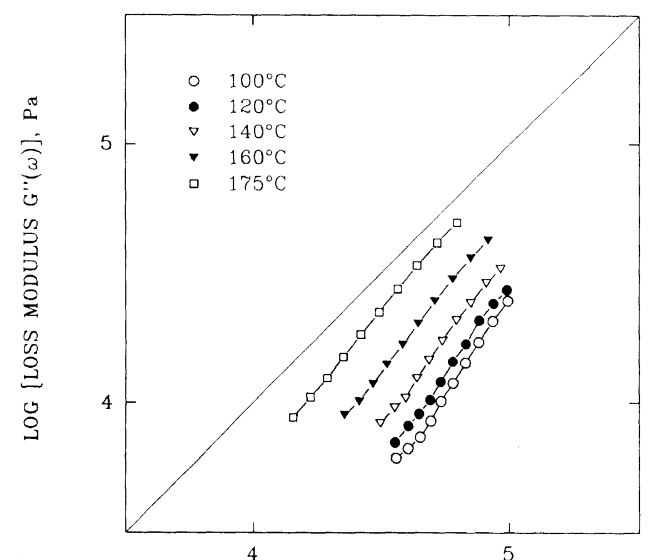

LOG [STORAGE MODULUS $\mathrm{G}^{\prime}(\omega)$ ], Pa

Figure 4. Plot of $\log G^{\prime \prime}(\omega)-\log G^{\prime}(\omega)$ for PVC/NBR-22 blend.

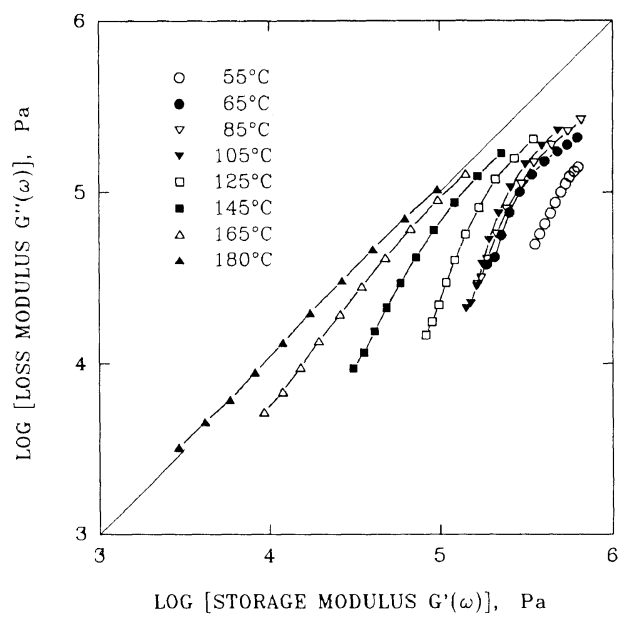

Figure 5. Plot of $\log G^{\prime \prime}(\omega)-\log G^{\prime}(\omega)$ for PVC/PCL blend.

equal values of $G^{\prime}(\omega)$ and $G^{\prime \prime}(\omega)$. It is shown that the $\log G^{\prime \prime}(\omega)-\log G^{\prime}(\omega)$ curves shift from the right to the left of the figure as the temperature increases. This temperature dependence of the $\log G^{\prime \prime}(\omega)-\log G^{\prime}(\omega)$ plots indicate some significant changes in the molecular behavior as well as phase morphology through the given temperature range. Of the two blends PVC/NBR-22, an incompatible system exhibits more noticeable shift to the left relative to PVC/NBR-30.

The $\log G^{\prime \prime}(\omega)-\log G^{\prime}(\omega)$ presentation of

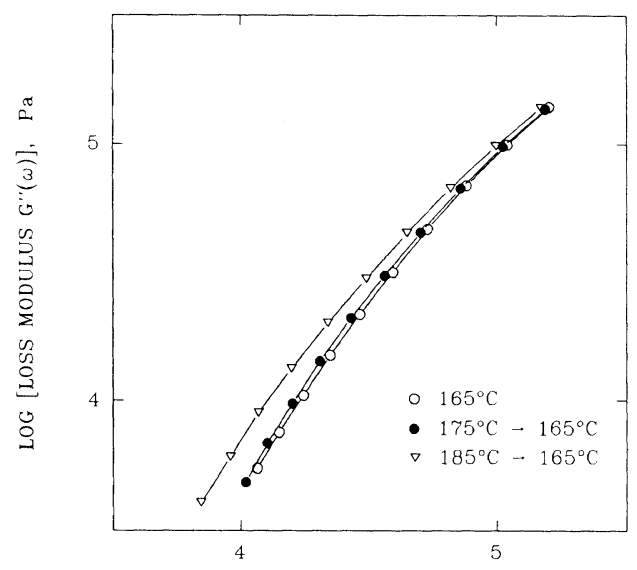

LOG [STORAGE MOdULUS $G^{\prime}(\omega)$ ], Pa

Figure 6. Effect of different heating history on PVC/PCL blend demonstrated by $\log G^{\prime \prime}(\omega)-\log G^{\prime}(\omega)$ plots.

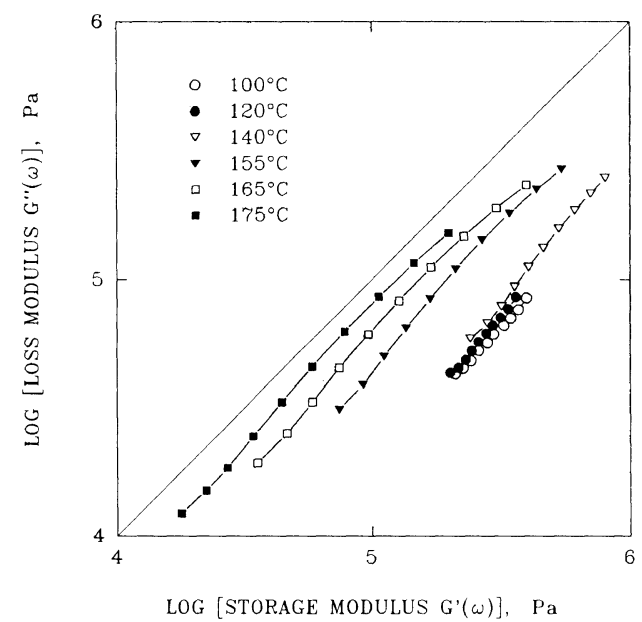

Figure 7. Plot of $\log G^{\prime \prime}(\omega)-\log G^{\prime}(\omega)$ for PVC/HYTREL blend.

PVC/PCL blend at various temperatures is given in Figur 5. In the temperature range between 60 and $105^{\circ} \mathrm{C}$, the curves do not move much to the left of the figure, but at the temperatures higher than $125^{\circ} \mathrm{C}$, the curves shift to the left much more significantly.

Figure 6 shows the effect of heating history on the viscoelastic properties and hence, on the $\log G^{\prime \prime}(\omega)-\log G^{\prime}(\omega)$ plots. When the PVC/PCL blend has been heated to $175^{\circ} \mathrm{C}$ and then cooled to $165^{\circ} \mathrm{C}$, the $\log G^{\prime \prime}(\omega)-\log G^{\prime}(\omega)$ plots shift to the left slightly. When heated to $185^{\circ} \mathrm{C}$ and 
then cooled to $165^{\circ} \mathrm{C}$, a more significant shift results.

In the $\log G^{\prime \prime}(\omega)-\log G^{\prime}(\omega)$ plots of PVC/HYTREL system, Figure 7 , the curves at $100^{\circ} \mathrm{C}$ and $120^{\circ} \mathrm{C}$ have almost the same position and shape. As the temperature increases, the curves shift to the left of the figure more significantly between 140 and $155^{\circ} \mathrm{C}$, and afterward moderately.

\section{DISCUSSION}

In the $\log G^{\prime \prime}(\omega)-\log G^{\prime}(\omega)$ plots, with reference to the equal-moduli line $\left(45^{\circ}\right.$ straight line), data positioned above the line indicate that the material behavior is dominated by the viscous response or loss modulus component. In contrast, data positioned below the line indicate the material behavior dominated by the elastic response. It was shown that all the $\log G^{\prime \prime}(\omega)-\log G^{\prime}(\omega)$ curves except for that of PVC/PCL blend at $180^{\circ} \mathrm{C}$ are positioned below the equal-moduli line, indicating that elastic behavior dominates at the respective temperatures. Also, the curves were shown to approach the equal-moduli line with increasing temperature, indicating that the material is becoming less elastic as the temperature is raised.

Two PVC/nitrile rubber blends (i.e., PVC/ NBR-30 and PVC/NBR-22) clearly demonstrate the temperature dependence of the $\log G^{\prime \prime}(\omega)-\log G^{\prime}(\omega)$ plots by displaying the shift of the curves from the right to the left of the figure. In a commercial PVC, distinct crystalline regions (i.e., microcrystallites) exist because of the presence of syndiotactic sequences of between 5 and 12 ordered monomer units ${ }^{14,15}$ along the chain, which are responsible for approximately $8-10 \%$ of crystallinity. ${ }^{16,17}$ It has been suggested that the PVC microcrystallites are not completely melted within normal processing temperature of PVC due to the wide melting range of $120-210^{\circ} \mathrm{C}^{18}$ As the microcrystallites melt, restrictions to molecular mobility decreases, and this results in the shift of the $\log G^{\prime \prime}(\omega)$ $\log G^{\prime}(\omega)$ plots to the left. In both systems, especially in PVC/NBR-22, the magnitude of the shift to the left is different at individual temperature range, which suggests that there are substantially differences in the amount of the microcrystallites corresponding to the individual temperature range.

Considering that the maximum chamber wall temperature of the mixing process was $165^{\circ} \mathrm{C}$ (see Table I), it is supposed that the microcrystallites whose melting range is at least below $165^{\circ} \mathrm{C}$ are melted completely during mixing. However, some shift of the $\log G^{\prime \prime}(\omega)-$ $\log G^{\prime}(\omega)$ curves indicates that some portion of the microcrystalline melts might be unaffected (unblended) and recrystallization of them must take place upon cooling. In contrast, the microcrystallites possessing the melting range higher than $165^{\circ} \mathrm{C}$ seem to be intact during mixing, which results in a relatively large shift of the $\log G^{\prime \prime}(\omega)-\log G^{\prime}(\omega)$ curves at the temperatures higher than $165^{\circ} \mathrm{C}$. In addition, the magnitude of the shift to the left is more significant for PVC/NBR-22 than for PVC/ NBR-30. This is because the former, an incompatible blend, retains more microcrystallites than the latter, a compatible one.

In Figure 5, the shift of the $\log G^{\prime \prime}(\omega)$ $\log G^{\prime}(\omega)$ plots of PVC/PCL system between 55 and $65^{\circ} \mathrm{C}$ is ascribed to the melting of crystalline PCL phase $\left(T_{\mathrm{m}} \mathrm{ca} .60^{\circ} \mathrm{C}\right)$; the further slight shift up to $150^{\circ} \mathrm{C}$ is caused by the softening of the PVC phase $\left(T_{\mathrm{g}} \mathrm{ca} .90^{\circ} \mathrm{C}\right)$. The heating to temperatures higher than $125^{\circ} \mathrm{C}$ reduces the crystallinity of PVC by melting and results in the shift of the curves to the left. At $180^{\circ} \mathrm{C}$, the $\log G^{\prime \prime}(\omega)-\log G^{\prime}(\omega)$ plot is slightly above the equal-moduli line, indicating that the system reaches the state where the viscous response just begins to dominate.

Figure 6 shows changes of the viscoelastic behavior caused by the different heating history. Heating to the higher temperature $\left(185^{\circ} \mathrm{C}\right)$ and then cooling to $165^{\circ} \mathrm{C}$ shift the curve to the left. Heating to $175^{\circ} \mathrm{C}$ and then cooling to $165^{\circ} \mathrm{C}$ results in a small shift. This 
indicates the melting of the microcrystallites at the higher temperature and incomplete recrystallization upon returning to the lower temperature. It also suggests that the maximum temperature of the material in the mixing operation was about $170-175^{\circ} \mathrm{C}$, somewhat higher than $160^{\circ} \mathrm{C}$ detected by the built-in thermocouple.

The $\log G^{\prime \prime}(\omega)-\log G^{\prime}(\omega)$ plots of PVC/ HYTREL blend shows a significant shift between 140 and $150^{\circ} \mathrm{C}$ because of the melting of the HYTREL crystallites $\left(T_{\mathrm{m}} \mathrm{ca} .150^{\circ} \mathrm{C}\right)$. The melting of the PVC microcrystallites must also be contributing to the shift.

\section{CONCLUSION}

Dynamic mechanical measurements were performed on four different model blends of PVC as a function of frequency at various temperatures.

The viscoelastic data measured at small strain in shear oscillation were represented in the form of $\log -\log$ plot of loss modulus $G^{\prime \prime}(\omega)$ against storage modulus $G^{\prime}(\omega)$. With reference to equal-moduli line ( $45^{\circ}$ straight line), all the blends were dominated by the elastic response at the respective temperatures, except for the PVC/PCL system at $180^{\circ} \mathrm{C}$. As the temperature increased, the $\log G^{\prime \prime}(\omega)-\log G^{\prime}(\omega)$ curves of all the blends shifted from the right to the left of the figure, indicating that the blends became less elastic. This was because of the melting of the PVC microcrystallites and melting of the crystallites of PVC/PCL and PVC/HYTREL blends. The effect of different heating history revealed through changes of the viscoelastic behavior made it possible to infer the actual maximum temperature of the material in the mixing operation.

Recognizing that the range of PVC microcrystallite melting is very broad (i.e., 120 $210^{\circ} \mathrm{C}$ ), it was concluded from the manner of the shift of the $\log G^{\prime \prime}(\omega)-\log G^{\prime}(\omega)$ curves that during mixing, some microcrystallites melted but others were intact, depending on their melting temperatures and then unblended portion of the microcrystalline melts recrystallized upon cooling, which resulted in the substantial differences in the amount of the microcrystallites in the resulting blends. In addition, the incompatible system was shown to retain more microcrystallites than the compatible one.

Acknowledgement. The author would like to thank Prof. N. Nakajima at Institute of Polymer Engineering, The University of Akron for his valuable suggestions and discussions.

\section{REFERENCES}

1. T. Murayama, "Dynamic Mechanical Analysis of Polymeric Materials," Elsevier Scientific, New York, 1978.

2. R. L. Hassel, Plastics Eng., 33, 37 (1977).

3. J. Heijboer, Polym. Eng. Sci., 19, 664 (1979).

4. N. Nakajima and E. R. Harrell, in "Current Topics in Polymer Science," Vol. II, R. M. Ottenbrite, L. A. Utracki, and S. Inoue, Ed., Hanser Publishers, New York, 1987.

5. K. S. Cole and R. H. Cole, J. Chem. Phys., 9, 341 (1941).

6. S. V. Kanakkanatt, J. Cell. Plast., Jan./Feb., 54 (1973).

7. W. W. Graessley and J. Roovers, Macromolecules, 12, 959 (1979).

8. C. D. Han and H-K. Chuang, J. Appl. Polym. Sci., 30, 4431 (1985).

9. L. A. Utracki, "Polymer Alloys and Blends," Hanser Puflisher, New York, 1990.

10. C. A. Daniels and E. A. Collins, J. Macromol. Sci.-Phys., B10, 287 (1974).

11. C. J. Ong, Ph. D. Thesis, University of Massachusetts, Amherst (1973).

12. R. J. Cella, Polym. Sci., Polym. Symp., 42, 727 (1973).

13. P. K. Freakley and W. V. Wan Idris, Rubber Chem. Technol., 52, 134 (1979).

14. G. Talamini and G. Vidotto, Macromol. Chem., 100, 48 (1967).

15. K. H. Hellwedge, U. Johnsen, and D. Kockott, Kolloid Z., 194, 5 (1964).

16. W. V. Titow, "PVC Plastics," Elsevier Applied Science, London, 1990.

17. C. Baker, W. F. Maddams, and J. Preedy, J. Polym. Sci., Polym. Phys. Ed., 15, 1041 (1977).

18. D. E. Witenhafer, J. Macormol. Sci.-Phys., 134, 915 (1970). 